\title{
Nuclear Morphology and the Biology of Cancer Cells
}

\author{
Edgar G. Fischer \\ Division of Surgical Pathology and Cytopathology, Department of Pathology, University of New Mexico, \\ Albuquerque, NM, USA
}

\section{Keywords}

Nuclear membrane irregularity · Cancer - Nuclear envelope . Signal transduction $\cdot$ Chromatin

\begin{abstract}
Background: For more than a century, diagnostic pathologists have used morphologic abnormalities of the nucleus as essential diagnostic features to distinguish benign from malignant cells. These features include nuclear enlargement and increased nuclear-to-cytoplasmic ratio, nuclear membrane irregularities, hyperchromasia, and abnormal chromatin distribution. As our knowledge about the genetic and epigenetic abnormalities of cancer cells has increased in recent decades, the pathophysiologic mechanisms that underlie these morphologic abnormalities remain incompletely understood. Summary: This review attempts to summarize biologic abnormalities in malignant cells related to these morphologic changes. The molecular anatomy of the nuclear envelope in normal and malignant cells is discussed as well as regulation of nuclear size and shape, regulation of signal transduction pathways by molecules of the nuclear envelope, chromatin distribution, and the effects of HPV infection on dysplastic cells in the uterine cervix. Key Message: Causes of morphologic nuclear abnormalities in malignant cells are likely multifactorial. They probably include muta-
\end{abstract}

tions, dysregulation of signal transduction pathways, abnormal gene expression patterns, alterations of nuclear envelope proteins and chromatin, and aneuploidy.

๑) 2020 S. Karger AG, Basel

\section{Introduction}

Morphologic abnormalities of the nucleus are key to establishing a diagnosis of malignancy in the daily practice of cytopathology and surgical pathology (Figs. 1,2). These features have been used by pathologists for many decades to distinguish benign from malignant cells and to render diagnostic conclusions that guide patient management. Early descriptions of morphologic abnormalities in malignant cells go back to the work of Lionel S. Beale in the 1860s [13]. In the cytopathology laboratory, specimens are scrutinized for cells with increased nuclear size, increase in nuclear-to-cytoplasmic ratio, morphologic irregularities of the nuclear membrane, and abnormal distribution of chromatin [4-8]. This is done for cervical Pap tests, body fluids such as pleural fluid or urine, and cells collected by fine needle aspiration biopsies. Malignant cells from various anatomic sites often display a combination, but not necessarily all of these morphologic abnormalities. The art and science of distinguishing between benign and malignant cells

$\begin{aligned} & \text { karger@karger.com } \\ & \text { www.karger.com/acy }\end{aligned}$
Karger ${ }^{\prime /}$



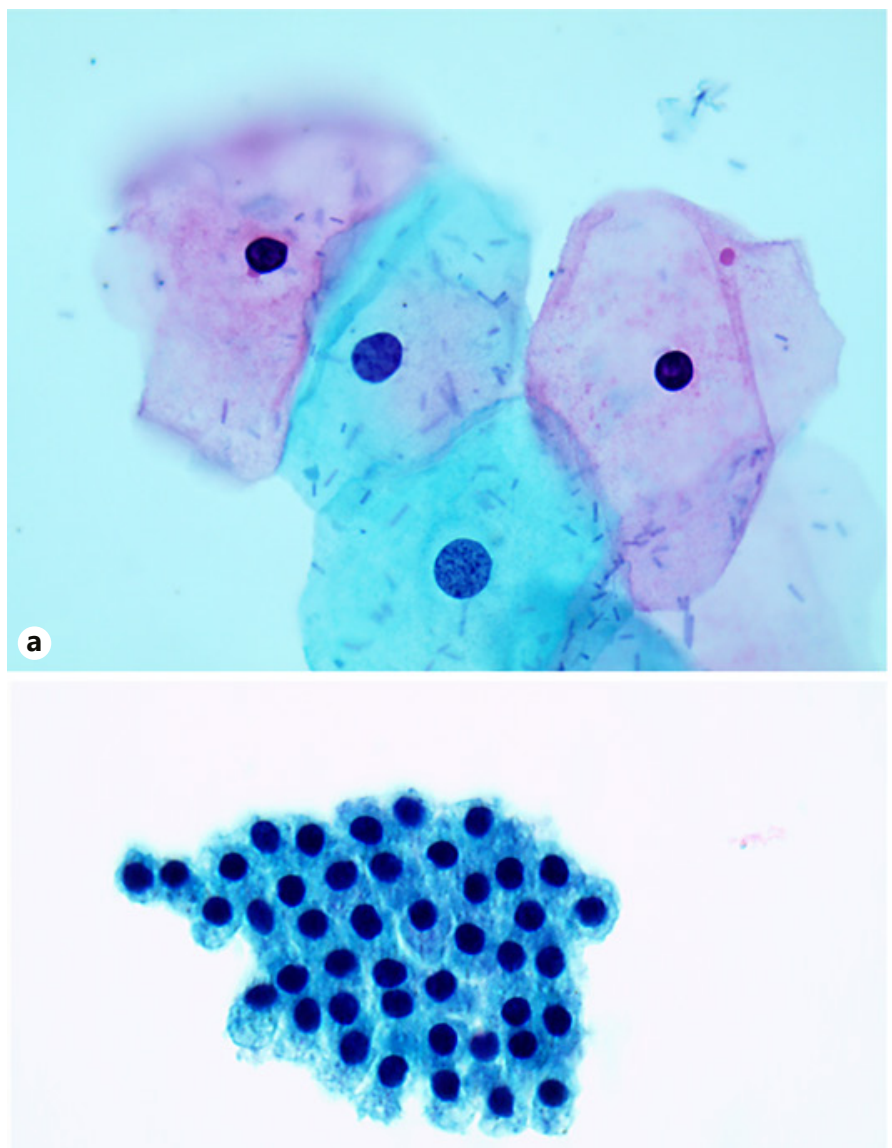

C

Fig. 1. a Squamous cells from a normal Pap test. Superficial cells have pink and intermediate cells have blue cytoplasm. Nuclei are small with round regular outlines. b Low-grade squamous dysplasia (CIN 1) in a Pap test. Nuclei are enlarged with coarse chromatin distribution. Nuclear membranes have irregular contours with folds and dents. Perinuclear koilocytic halos are seen. c Normal bile duct epithelial cells from benign bile duct brushings. Nuclei

rests on morphologic features of the nucleus, although other findings such as specimen cellularity are also important diagnostic considerations. Nuclear membrane irregularities include thickening, dents, folds, grooves, and pseudoinclusions. Chromatin is abnormally distributed in malignant cells and is often coarse or vesicular, or can be delicate and more evenly distributed as in small cell neuroendocrine carcinomas. These cytomorphologic features are also important in the evaluation of tissue biopsies, where they are assessed in conjunction with architectural features such as
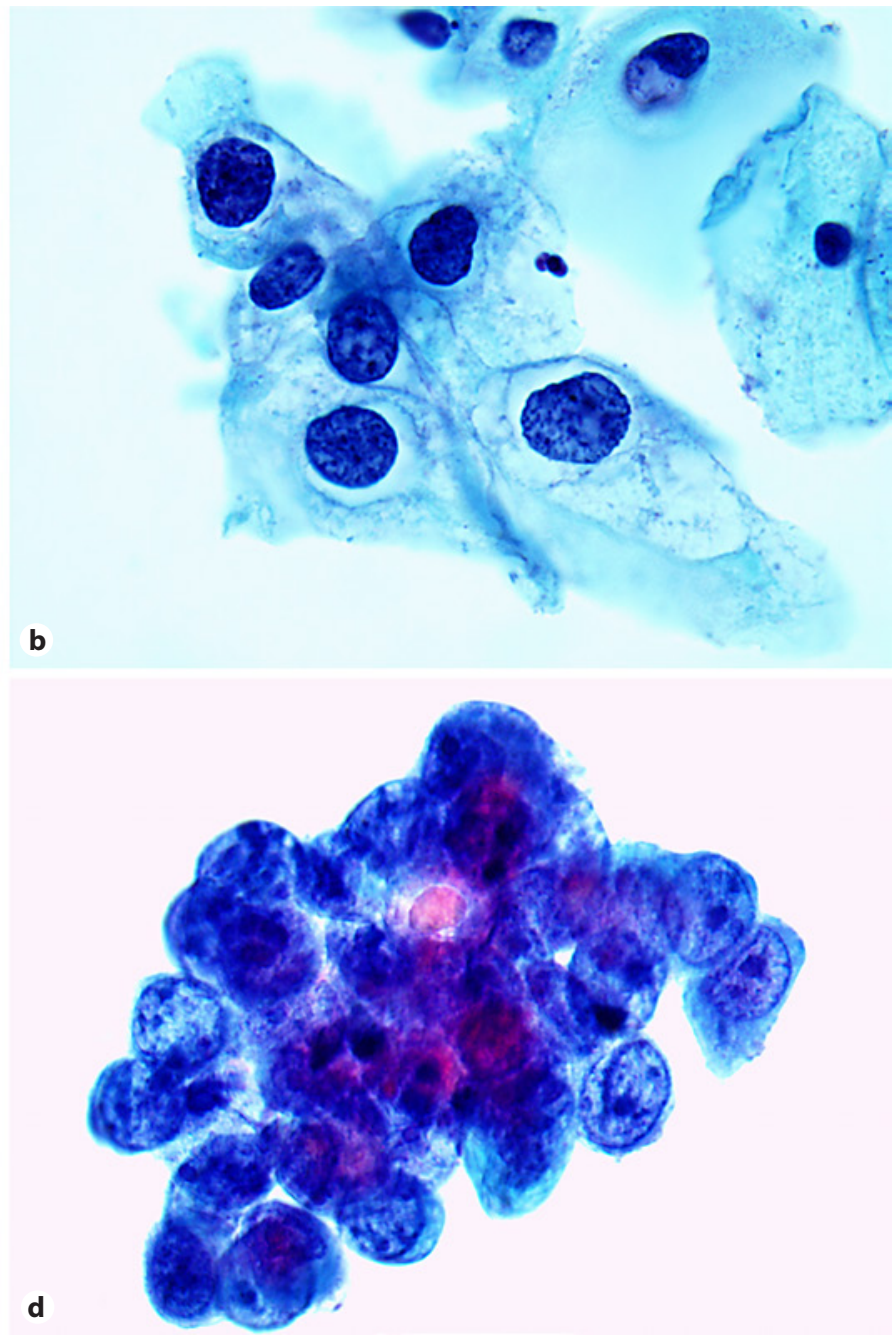

are small and evenly spaced. They are round with regular contours. d Malignant cells of a cholangiocarcinoma from bile duct brushings. Nuclei are markedly enlarged and overlapping. They have thickened nuclear membranes, irregular contours, and prominent nucleoli. All images were taken at the same magnification of $\times 1,000$ with an oil immersion objective to allow for comparison of nuclear size and shape.

the growth pattern of neoplastic cells and the presence of a desmoplastic stromal response.

Over the past 20-30 years, our knowledge about cancer biology has expanded dramatically, most recently with the accelerated discovery of molecular genetic and epigenetic abnormalities of cancer cells. This includes our current understanding of the abnormal nuclear structure and function of malignant cells and their underlying biologic mechanisms. The structural organization of the normal mammalian nucleus is well established. The 

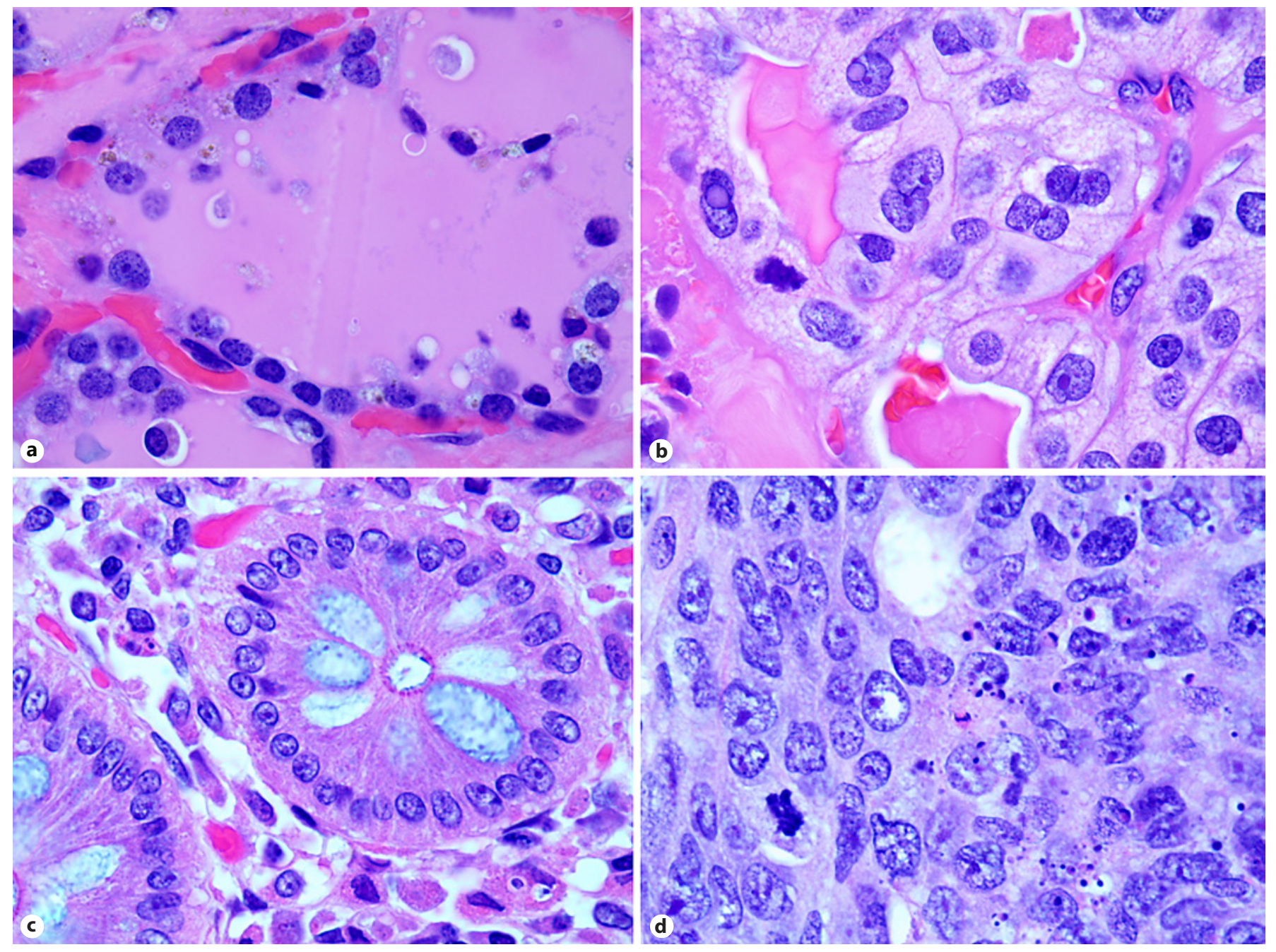

Fig. 2. a Normal thyroid follicle. Nuclei are small and round. b Papillary thyroid carcinoma. Nuclei are enlarged with irregular nuclear contours including folds and dents in the nuclear membranes. Two nuclei have pseudo-inclusions highly characteristic of papillary thyroid carcinoma. Nucleoli are prominent. c Normal crypts in the colon. Nuclei are small and mostly round. d High-

structural and functional abnormalities of malignant nuclei are increasingly coming to light. Pathomechanisms behind these abnormalities are emerging, even though many key questions remain unanswered. This review attempts to summarize how genetic and epigenetic abnormalities of cancer cells influence nuclear morphologic abnormalities. It also explores how microscopically visible nuclear abnormalities impact the biology of malignant cells. As our knowledge expands, the convergence between morphologic features and the underlying genetic and pathophysiologic features of cancer cells will lead to

grade colonic adenocarcinoma with large nuclei, highly irregular nuclear contours with folds and dents, and coarse chromatin. A mitotic figure is present. All images were taken at the same magnification of $\times 1,000$ with an oil immersion objective to allow for comparison of nuclear size and shape.

a better understanding of the relationship between abnormal structure and function and the biology of cancer.

\section{Molecular Anatomy of the Nuclear Envelope}

Nuclear membrane irregularities are one of the key morphologic features of malignant cells. The nuclear membrane is part of the nuclear envelope (NE) that separates the nucleus from the cytoplasm (Fig. 3). The NE is a complex compartment comprising a membrane system 
Fig. 3. Schematic overview of the NE and chromatin. The NE is composed of the INM (black), the ONM (black), and the nuclear lamina (purple). NPCs (green) connect the nuclear interior to the cytoplasm. The INM is connected to the nuclear lamina via INM proteins (red), and ONM proteins (yellow) are connections to the cytoskeleton. INM proteins can bind to chromatin (blue) via linker proteins (orange). The ONM is contiguous with the endoplasmatic reticulum (not shown). NE, nuclear envelope; INM, inner nuclear membrane; ONM, outer nuclear membrane; NPC, nuclear pore complex.

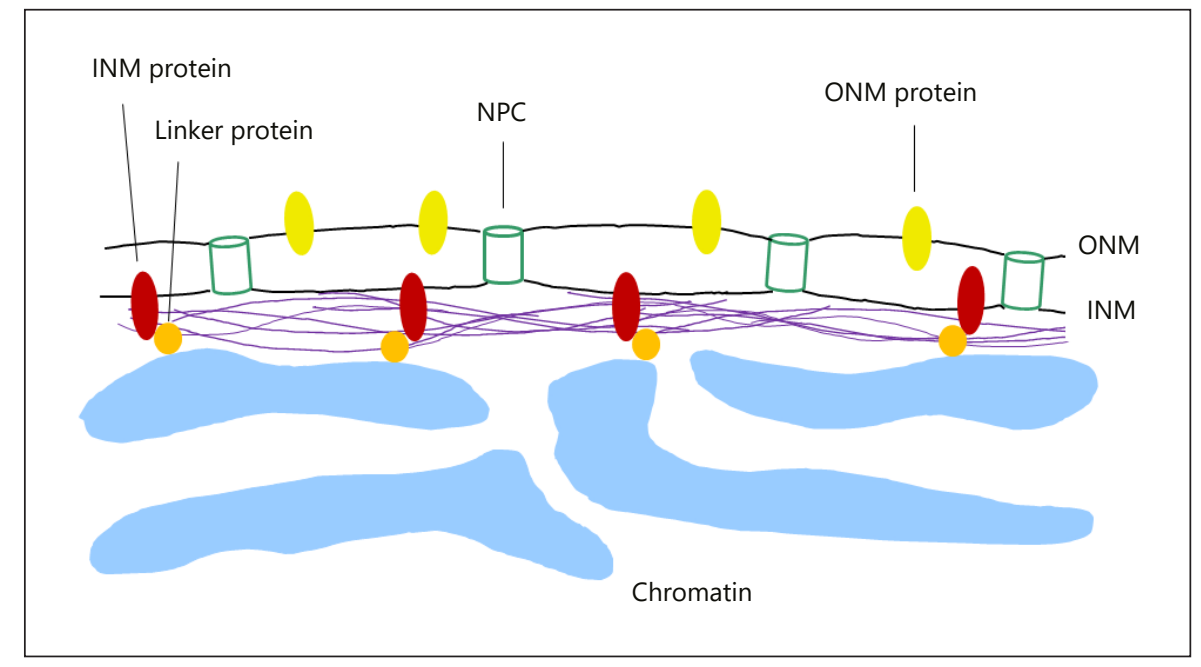

of 2 phospholipid bilayers, the outer (ONM) and inner nuclear membrane (INM), and the nuclear lamina. The ONM and INM are joined at nuclear pore complexes (NPCs) that are inserted into the phospholipid bilayers. NPCs are very large (>60 MDa in mammalian cells) protein complexes that regulate the directional transport of soluble molecules between the nucleus and the cytoplasm [9-11], and nucleoporins are one of their key components $[4,12]$. Between the ONM and INM is a $30-50-\mathrm{nm}$ wide perinuclear space that is contiguous with the space of the rough endoplasmatic reticulum.

Proteins in the ONM build connections to the cytoskeleton that anchor the nucleus within the cell. Located under the INM is the nuclear lamina that faces the interior of the nucleus, the nucleoplasm. The nuclear lamina is primarily composed of lamin proteins, which are intermediate filaments that form a polymeric meshwork and provide structural and tensile integrity to the nucleus. Lamin A/C (A-type lamins) are the product of alternative splicing of the $L M N A$ gene, while lamin $\mathrm{B} 1$ and $\mathrm{B} 2$ (Btype lamins) are encoded by $L M N B 1$ and $L M N B 2$ genes, respectively $[4,13]$. The lamin polymer meshwork is connected to the INM via inner nuclear envelope transmembrane proteins (NETs), and SUN proteins are the main inner NETs. Toward the nuclear interior, the nuclear lamina provides structural connections to chromatin. Lamins, many NETs, and some NPC proteins bind directly to chromatin $[4,9-11]$. NETs have been subject to intense investigation. Close to 1,000 NETs have been identified, and many of them are preferentially expressed in certain tissue types $[1,14]$. Some inner NETs that bind the lamin polymer connect to outer NETs, which are con- nected to the cytoskeleton. The main inner NETs are SUN proteins, and the main outer NETs are nesprins/ syne proteins. Together, inner NETs (SUN proteins) and outer NETs (nesprins) form the LINC (linker of nucleoskeleton and cytoskeleton complex) $[9,10]$. Taken together, the NE plays a central and critical role in supporting nuclear shape and stability by anchoring the nucleus within the cell. By providing docking sites for chromatin, the NE contributes to tissue-specific genome organization and regulation of gene expression. As described below, the NE is involved in regulating signal transduction pathways. It is quite intriguing to think about these biologic functions and their dysregulation when examining malignant cells under the microscope in diagnostic pathology.

\section{Regulation of Nuclear Size}

Nuclear enlargement is one of several key morphologic changes in malignant cells, but also a characteristic change of benign reactive cells in response to inflammatory or other injury. The pathophysiologic mechanisms that underlie increase in nuclear size in malignant cells remain largely unknown $[15,16]$. It is well established that nuclear size is tightly regulated. Across species and cell types, normal cells tend to maintain a roughly constant ratio of nuclear volume to cell volume. In normal cells, nuclear size tends to scale with cell size [16], thus maintaining a constant ratio often referred to as nuclearto-cytoplasmic ratio by diagnostic pathologists and as karyoplasmic ratio in the experimental biology literature. 
Ploidy correlates with nuclear size in experimental model systems in many species including Xenopus, Tetrahymena, Drosophila, plants, and mice and in mammalian cell culture systems [16]. On the other hand, aneuploidy is common is many human malignancies. Highly aggressive malignancies such as high-grade carcinomas, sarcomas, and lymphomas often have a much higher level of aneuploidy than lower grade tumors and typically have larger nuclei and more pronounced abnormalities in nuclear shape. Some malignancies exhibit nuclear morphologic abnormalities while retaining diploidy. A lack of correlation between nuclear size and ploidy has been reported for carcinomas of the bladder, colon, breast, lung, skin, cervix, and prostate [16]. Therefore, the level of ploidy cannot be the only determinant of nuclear size. There is also a strong correlation between genome size and cell size. Organisms with larger genomes tend to have larger cells and larger nuclei. This conclusion is supported by an abundance of data across eukaryotic organisms that span 5 orders of magnitude in terms of genome content [16]. At the same time, different cell types with their diverse cellular functions within the same organism have differences in cell and nuclear size while sharing the same genome. But these general correlations strongly suggest that genome size and ploidy are an important determinant of nuclear size [16].

Besides aneuploidy and genomic alterations in malignant cells, abnormalities in the structural components of the NE likely influence nuclear size. Lamins, the main components of the nuclear lamina that structurally supports the nuclear membrane, are aberrantly expressed in many human tumors. Abnormal expression of lamin isoforms have been reported in multiple studies and vary depending on the subtype of cancer and other biological properties such as tumor grade [16-18]. There is also growing evidence that LINC complexes are important for nuclear size regulation. These complexes consist of protein families that connect the nuclear lamina to the cytoskeleton through the NE. These connections are mediated by interactions between inner nuclear membrane SUN-domain containing proteins and outer nuclear membrane proteins such as nesprins, which directly or indirectly bind actin. LINC complexes perform diverse cellular functions including in cell division, regulating nucleus-centrosome attachments, and nuclear migration and anchorage [16].

The specific molecular alterations that underlie nuclear enlargement and intratumoral nuclear size variation are largely unresolved. As nuclear enlargement in malignant cells is often associated with changes in nuclear shape, nuclear membrane irregularities, and changes in chromatin distribution, nuclear size regulation cannot be considered in isolation [16, 17]. One intriguing hypothesis is that nuclear enlargement may be manifested in abnormal nuclear shapes as attempts to maintain a constant nuclear-to-cytoplasmic ratio. The subsequent nuclear shape changes may then lead to altered nuclear architecture, altered chromatin organization, and altered functional states, including the altered gene expression that occurs during carcinogenesis and tumor progression [16, 17].

\section{Regulation of Nuclear Shape}

Nuclei of most normal epithelial cells are round to ovoid, most lymphoid cells have round nuclei (with germinal center cells as one exception), and stromal cells have ovoid to spindle-shaped nuclei, all generally with smooth nuclear outlines. Reactive mesothelial cells are an exception as they can have nuclear folds in cytology specimens of peritoneal washings. Many malignancies have irregularities in nuclear contours including dents, folds, grooves, and in some cases pseudo-inclusions. But the pathophysiologic mechanisms that underlie these shape abnormalities have not been elucidated.

Some tumors with prominent nuclear membrane irregularities have defined molecular alterations, but there is no known connection between the morphologic and genetic changes. The most prominent example is papillary thyroid carcinoma (PTC) with nuclear grooves and pseudo-inclusions as their morphologic hallmarks (Fig. 2b). About $45 \%$ of PTCs have BRAF V600E mutations, about $20 \%$ have RAS mutations, and smaller subsets have RET-PTC rearrangements that generate a chimeric tyrosine kinase, or $P A X 8-P P A R-\gamma$ rearrangements. PTCs typically have a diploid karyotype [19]. An elegant study where normal thyroid epithelial cells were microinjected with the RET-PTC oncogene showed that within hours cells developed nuclear membrane irregularities. This occurred without cells going through mitosis, indicating that nuclear membrane irregularities can develop without postmitotic nuclear assembly [20]. PTCs also commonly have nuclear pseudo-inclusions, complex invaginations of the nuclear membrane into the nucleus. Nuclear pseudo-inclusions can also be found in some lung adenocarcinomas, particularly in tumors with EGFR mutations, malignant melanomas, and hepatocellular carcinomas $[8,21]$. Malignant melanomas and benign melanocytic nevi often have nuclear pseudo-inclusions, 
and about $50 \%$ of melanomas and over $50 \%$ of benign nevi also have BRAF mutations. Therefore, both pseudoinclusions and $B R A F$ mutations are also found in benign lesions and are not necessarily associated with malignant behavior. Longitudinal nuclear grooves are a diagnostic hallmark of Langerhans cell histiocytosis, and about $60 \%$ of cases have BRAF mutations [6]. The biologic behavior of this disease ranges from benign to malignant and is highly dependent on the clinical setting such as disease location and patient age. Adult ovarian granulosa cell tumors are characterized by longitudinal nuclear grooves that impart a coffee been shape. These sex cord stromal tumors have a characteristic point mutation in the FOXL2 gene in an otherwise mutation-free background. The biologic behavior of adult granulosa cell tumors is largely unpredictable, with neoplastic spread occurring in a subset of patients. Therefore, nuclear shape irregularities are found in a variety of malignancies and in some low-grade and benign neoplasms. There is no definitive association of these irregularities with specific molecular alterations, and the underlying molecular pathomechanisms for these morphologic changes remain unknown.

\section{NE Protein Expression Is Altered in Many Human Tumors}

Given that nuclear membrane irregularities are a common morphologic finding in malignant cells, many studies have focused on the abnormal expression of proteins in the NE. Expression patterns of lamins and other NE proteins have been investigated in several human malignancies. Reported findings vary widely among tumor types, and even within the same tumor, depending on study methods and antibodies used. For colon cancers, both increased and decreased levels of lamin A/C expression have been reported. Furthermore, lamin A/C expression levels were found to be highly heterogeneous within single tumors. Depending on the individual study, both high and low levels of lamin A/C have been considered poor prognostic markers. Loss or reduced levels of lamin $\mathrm{A} / \mathrm{C}$ in ovarian cancers were found in some studies [18] while elevated levels were in detected in others $[15,18]$. Lamin A/C expression was strikingly reduced or absent in most small cell lung cancers, but preserved in pulmonary squamous cell carcinomas and adenocarcinomas [22]. The majority of cutaneous squamous cell carcinomas were strongly positive for A-type lamins, and most tumors with reduced expression were poorly differentiated. Most studies of basal cell carcinomas found reduc- tion of A-type lamins [16]. Lamin A/C overexpression was also found in ovarian and prostate cancers and correlated with adverse prognostic factors, while emerin expression was reduced in ovarian tumors [22]. B-type lamin expression was reduced in some carcinomas of the lung and intestinal tract and cutaneous squamous cell carcinomas. Lamin B1 expression was found frequently elevated in hepatocellular carcinomas and prostate cancers [16]. Lamin levels and localization patterns have been proposed as potential biomarkers for diagnosis and prognosis of different cancers. Immunofluorescence staining for lamin B and emerin was used to assess nuclear pleomorphism in breast cancer and found to be superior to traditional histologic grading procedures $[16,17$, 23]. Abnormal expression of NETs have also be found in several tumor types, but gene expression microarrays showed inconsistent levels of dysregulation for many genes. One study found the NET gene LPCAT3 strongly upregulated in ovarian cancers [1].

\section{NE Molecules Are Involved in the Regulation of Signal Transduction Pathways and Cell Migration}

The molecular mechanisms that cause abnormalities in nuclear size and shape in malignant cells remain largely unresolved. But it has emerged that NE abnormalities in malignant cells influence a broad spectrum of cellular functions, including signal transduction pathways, regulation of the cell cycle, apoptosis, and genomic stability. The NE structurally and functionally connects the cytoskeleton to the nucleoskeleton, and it is emerging as a nexus for signal transduction between the cytoplasm and the nucleus $[1,4,10,13,14,24]$. Lamins are the main NE structural proteins, and NETs have been shown to interact with signal transduction pathways. Lamin A and several NETs including LAP2 $\alpha$ (lamin-associated polypeptide $2 \alpha$ ) sequester proteins such as the tumor suppressor retinoblastoma protein $\mathrm{pRb}$ away from its target genes, which likely influences cell proliferation $[4,25]$. Lamins $\mathrm{A} / \mathrm{C}$ and their binding partner LAP $2 \alpha$ have been reported to interact with $\mathrm{pRb}$, repress $\mathrm{E} 2 \mathrm{~F}$-dependant transcription, and promote cell cycle arrest. Loss of lamin A/C or LAP $2 \alpha$ impairs normal cell cycle regulation, leading to inappropriate S-phase entry [26]. Lamin A has been connected to the MAPK signaling pathway, and alterations in A-type lamins have been linked to the PI3K-AKTmTOR pathway $[4,13]$. Increased lamin $\mathrm{A} / \mathrm{C}$ levels in prostate cancer cause changes in the PI3K-AKT-mTOR pathway, and lamin $\mathrm{A} / \mathrm{C}$ upregulation in colorectal can- 
cer induces changes in cytoskeletal organization that promote cell motility $[4,15]$. Lamin A also interacts with the nuclear envelope transmembrane (NET) protein MAN1 and regulates receptor-mediated smads, which are intracellular mediators of the TGF- $\beta$ cytokine family [13]. Emerin is an NET that can bind $\beta$-catenin and thus regulate the $\beta$-catenin-Wnt signaling pathway $[13,14]$. Since some NETs can directly interact with tumor suppressors and many NETs are expressed in a tissue-specific manner, future investigations may uncover possible mechanisms that could be specific to certain tumor types [14]. Nesprins are outer NETs and also have a role in signal transduction, with possible implications in oncogenesis [27]. The SYNE1 gene that encodes nesprin 1 was found mutated in 5 of 11 colorectal cancers, whereas a different study found the SYNE1 gene promotor methylated in all of 102 colorectal cancers. Nesprin 1 expression was downregulated in a majority of ovarian and breast cancers. The SYNE2 gene that encodes nesprin 2 was found altered in a subset of breast cancers [16]. Abnormal nuclear lamina organization can also lead to apoptosis, and lamins are targets for proteolytic degradation by caspases [28, 29]. Overall, the nuclear lamina has a broad range of functions in regulating cellular processes that include signal transduction, gene expression, DNA repair, and cellular senescence, which all await further elucidation [13].

Cell migration is a key cellular function in the dissemination of malignant cells. During migration, cells are exposed to the biomechanical forces in their microenvironment and have to pass through narrow constrictions in the extracellular matrix and through barriers posed by other cells $[4,24,30,31]$. The nucleus is the largest and stiffest organelle of the cell and dominates the biomechanical process of migration. The nuclear lamina and nuclear interior influence the mechanical deformability of the nucleus [4]. Biomechanical forces from the extracellular matrix are transmitted from the cell surface into the cell through focal adhesion complexes and the actin-myosin cytoskeleton to the NE $[4,24,30,31]$. NE molecules including lamins and LINC complexes are involved in the transmission of biomechanical forces from the cytoskeleton to the nucleoskeleton [24]. The LINC components involved in these processes include the inner NETs (SUN proteins) and outer NETs (nesprins). Therefore, changes in the composition of the NE and in the nuclear architecture may modify the rigidity of the nucleus, alter chromatin structure and gene expression, and facilitate migration and dissemination of malignant cells [4, 24, 30, 31].

Nuclear Morphology and Cancer Biology

\section{Biologic Implications of Chromatin Distribution in Malignant Cells}

In the practice of cytopathology and histopathology, the morphology of malignant nuclei is typically described as hyperchromatic with coarse or clumped chromatin distribution and in some cases as vesicular or open $[5,6$, 8]. These morphologic features likely correlate with profound alterations in chromatin structure and function, with resultant changes in gene expression states and/or chromosome stability [32]. Changes in chromatin texture are probably caused by condensation or decondensation of chromatin domains [8]. Studies in culture cells found that experimental expression of activated HRAS can induce chromatin coarsening, but the underlying mechanisms are unknown [8].

On a molecular level, chromatin is the complex that DNA forms with histone proteins to organize and package DNA. DNA is wrapped around histone proteins to form nucleosomes [33]. The protein components of chromatin play a critical role in regulating gene expression. Chromatin exists in 2 forms: (1) heterochromatin, which is densely packed, transcriptionally silent, and located at the nuclear periphery, and (2) euchromatin, which is more dispersed, transcriptionally active, and located in the more central part of the nucleus. Chromatin remodeling is the dynamic modification of chromatin architecture that allows the transcriptional machinery proteins access to genomic DNA and thereby control gene expression. This is principally carried out by covalent histone modifications by specific enzymes, including histone acetyltransferases, methyltransferases, and others.

Linking morphologic nuclear changes of cancer cells to specific molecular alterations is one of the great challenges in cancer research, and epigenetic changes likely are a key component of this link [32]. The study of epigenetic changes in cancer cells, that is, the study of molecular changes that do not involve alterations in DNA sequence, has been an increasingly important focus in recent years. Key epigenetic changes in malignant cells include changes in DNA methylation, histone modifications, and changes in the composition of nucleosomes [32]. These epigenetic modulations of chromatin are associated with changes in gene expression patterns $[8,32]$. Molecules of the NE including lamins and many NETs and NPCs serve as anchoring sites for chromatin [9-11, 34]. A- and B-type lamins bind to chromatin through interactions with INM proteins containing the LAP2-emerin-MAN1 domain [12]. Lamin B1 is mainly associated with heterochromatin [12]. Many of these molecules in-

Acta Cytologica 2020;64:511-519

DOI: $10.1159 / 000508780$ 
fluence heterochromatin assembly at the NE and can have an effect on gene positioning and gene expression [35]. In a model illustrated by Stancheva and Schirmer [9], transcriptionally inactive peripheral heterochromatin tends to be associated with lamins and NETs of the INM. The NETs and nuclear lamina recruit chromatin that has been modified with specific epigenetic marks, provide silencing factors that add new epigenetic modifications to genes located at the nuclear periphery, and sequester transcription factors away from the nuclear interior. Some chromatin-binding partners of NETs specifically reflect transcriptionally silenced chromatin [12]. On the other hand, transcriptionally active peripheral euchromatin tends to be associated with NPCs. Proteins of the nuclear pores bind active euchromatin, promote transcription, and coordinate gene expression with mRNA transport [9]. The microtubule organizer $\gamma$-tubulin has been found throughout the chromatin and may play a distinct role in promoting NE assembly during formation of the nucleus after cell division [4]. In addition to the NE abnormalities found in malignant cells, it is well accepted that A- and B-type lamins are altered during the aging process. NE regions where lamins are decreased are more predisposed to NE alterations such as bleb formation and rupture [12]. NPCs including nucleoporins as their key components are also thought to be involved in the pathogenesis of aging and of aging-associated diseases [12].

\section{HPV Infection and Nuclear Morphologic Abnormalities in Cervical Dysplasia}

HPV infection is the key etiologic factor for the development of cervical squamous dysplasia and carcinoma. Infection by high-risk viruses (e.g., HPV-16 and -18) is essential but not sufficient for malignant transformation, but our knowledge of viral oncogenesis in the cervix remains incomplete. Following chronic infection, expression of the viral oncogenes E6 and E7 are critical for this transformation as their corresponding proteins inactivate the tumor suppressors $\mathrm{Rb}$ and $\mathrm{p} 53$, respectively, activate cyclins, and inhibit apoptosis $[36,37]$. Integration of viral DNA into the host also leads to genomic instability [18, 38]. Nuclear morphologic abnormalities are now linked to the mechanisms of viral carcinogenesis. Early reports indicate that activation of oncogenes or inactivation of tumor suppressor genes leads to nuclear morphologic changes, but the underlying molecular mechanisms remain unresolved [18]. It is clear that persistent HPV infection causes disruption of the nuclear lamina, which is visible as a change in nuclear morphology on the Pap test (Fig. 1b) [18]. Expression of lamin A/C by immunohistochemistry in cervical dysplasias is heterogeneous and highly variable. One study found that lamin A/C expression was lost in $70 \%$ of cervical cancer tissues, and patchy and abnormal distributed lamin A/C staining in the other $30 \%$ of cases. As integration of the HPV genome into the host cell is random, these findings invite speculation about correlation with integration of the viral genome [22]. Therefore, altered expression of nuclear lamina proteins likely contributes to abnormal nuclear morphology. Persistent HPV infection likely leads to disruption of the nuclear lamina and accounts for changes in nuclear morphology [18].

\section{Conclusions}

As diagnostic pathologists use morphological abnormalities of the nucleus for the diagnosis of malignancy, the underlying molecular mechanisms remain unclear. On the other hand, many recent advances in cancer research are beginning to shed light on these mechanisms, and our knowledge is expanding rapidly. The molecular anatomy of the NE of normal cells is well known. Nuclear size and shape are tightly regulated in eukaryotic cells. Several neoplasms including PTC, Langerhans cell histiocytosis, and some melanomas have nuclear grooves and/ or pseudo-inclusions, and a subset of these lesions also have BRAF V600E mutations. But, there is no known direct relationship between defined mutations and nuclear membrane irregularities. Aneuploidy is common in highgrade malignancies, which tend to have larger nuclei and more pronounced nuclear membrane irregularities.

Expression of NE proteins such as lamins is altered in a broad spectrum of carcinomas. NE abnormalities influence cellular functions including cell cycle, apoptosis, and genomic stability, with the NE emerging as a nexus for signal transduction pathways between the cytoplasm and the nucleus. Migration is an important cellular function in the dissemination of malignant cells. Biomechanical forces from the extracellular environment are transmitted to the nucleus through NE components including lamins and LINC complexes. They influence the mechanical deformability of the nucleus, the largest and stiffest organelle in the cell. Many NE proteins interact with chromatin and chromatin-associated proteins and influence transcription and gene expression patterns. In cervical dysplasia, HPV infection leads to changes in nuclear size and shape. Integration of HPV DNA into host cells 
leads to disruption of the nuclear lamina and to genomic instability. Causes of nuclear membrane irregularities are likely multifactorial and may include mutations, dysregulation of signal transduction pathways, abnormal gene expression patterns, alterations of NE proteins and chromatin, and aneuploidy.

\section{Disclosure Statement}

The author has no conflicts of interest to declare.

\section{Funding Sources}

The author did not receive any funding.

\section{Author Contributions}

The study conception and design, writing of the manuscript, and preparation of images were all done by the author.

\section{References}

1 de Las Heras JI, Schirmer EC. The nuclear envelope and cancer: a diagnostic perspective and historical overview. Adv Exp Med Biol. 2014;773:5-26.

2 Long SR, Cohen MB. Classics in cytology. VI: the early cytologic discoveries of Lionel S. Beale. Diagn Cytopathol 1993;9(5):595-8.

3 Naylor B. The century for cytopathology. Acta Cytol. 2000;44(5):709-25.

4 Alvarado-Kristensson M, Rosselló CA. The biology of the nuclear envelope and its implications in cancer biology. Int J Mol Sci. 2019; 20(10):2586.

5 Dey P. Chromatin pattern alteration in malignant cells: an enigma. Diagn Cytopathol. 2005;32(1):25-30.

6 Dey P. Cancer nucleus: morphology and beyond. Diagn Cytopathol. 2010;38(5):382-90.

7 Fischer AH, Zhao C, Li QK, Gustafson KS, Eltoum IE, Tambouret R, et al. The cytologic criteria of malignancy. J Cell Biochem. 2010; 110(4):795-811.

8 Zink D, Fischer AH, Nickerson JA. Nuclear structure in cancer cells. Nat Rev Cancer. 2004;4(9):677-87.

9 Stancheva I, Schirmer EC. Nuclear envelope: connecting structural genome organization to regulation of gene expression. Adv Exp Med Biol. 2014;773:209-44.

10 Stewart CL, Roux KJ, Burke B. Blurring the boundary: the nuclear envelope extends its reach. Science. 2007;318(5855):1408-12.

11 Webster M, Witkin KL, Cohen-Fix O. Sizing up the nucleus: nuclear shape, size and nuclear-envelope assembly. J Cell Sci. 2009;122(Pt 10):1477-86.

12 Martins F, Sousa J, Pereira CD, da Cruz E Silva $\mathrm{OAB}$, Rebelo $\mathrm{S}$. Nuclear envelope dysfunction and its contribution to the aging process. Aging Cell. 2020:19(5):e13143.

13 Choi JC, Worman HJ. Nuclear envelope regulation of signaling cascades. Adv Exp Med Biol. 2014;773:187-206.

14 Robson MI, Le Thanh P, Schirmer EC. NETs and cell cycle regulation. Adv Exp Med Biol. 2014;773:165-85.
15 Denais C, Lammerding J. Nuclear mechanics in cancer. Adv Exp Med Biol. 2014;773:43570.

16 Jevtic P, Levy DL. Mechanisms of nuclear size regulation in model systems and cancer. Adv Exp Med Biol. 2014;773:537-69.

17 Chow KH, Factor RE, Ullman KS. The nuclear envelope environment and its cancer connections. Nat Rev Cancer. 2012;12(3):196209.

18 Smith ER, George SH, Kobetz E, Xu XX. New biological research and understanding of $\mathrm{Pa}$ panicolaou's test. Diagn Cytopathol. 2018; 46(6):507-15

19 Acquaviva G, Visani M, Repaci A, Rhoden KJ de Biase D, Pession A, et al. Molecular pathology of thyroid tumours of follicular cells: a review of genetic alterations and their clinicopathological relevance. Histopathology. 2018; 72(1):6-31.

20 Fischer AH, Taysavang P, Jhiang SM. Nuclear envelope irregularity is induced by RET/PTC during interphase. Am J Pathol. 2003;163(3): 1091-100.

21 Fischer AH. The diagnostic pathology of the nuclear envelope in human cancers. Adv Exp Med Biol. 2014;773:49-75.

22 Broers JL, Ramaekers FC. The role of the nuclear lamina in cancer and apoptosis. Adv Exp Med Biol. 2014;773:27-48.

23 Bussolati G, Maletta F, Asioli S, Annaratone L, Sapino A, Marchiò C. "To be or not to be in a good shape": diagnostic and clinical value of nuclear shape irregularities in thyroid and breast cancer. Adv Exp Med Biol. 2014;773: $101-21$.

24 Tingey M, Mudumbi KC, Schirmer EC, Yang W. Casting a wider net: differentiating between inner nuclear envelope and outer nuclear envelope transmembrane proteins. Int J Mol Sci. 2019;20(21):5248.

25 Brachner A, Foisner R. Lamina-associated polypeptide (LAP) $2 \alpha$ and other LEM proteins in cancer biology. Adv Exp Med Biol. 2014; 773:143-63

26 Kennedy BK, Pennypacker JK. RB and lamins in cell cycle regulation and aging. Adv Exp Med Biol. 2014;773:127-42.
27 Jevtic P, Edens LJ, Vukovic LD, Levy DL. Sizing and shaping the nucleus: mechanisms and significance. Curr Opin Cell Biol. 2014;28: 16-27.

28 Broers JL, Machiels BM, Kuijpers HJ, Smedts F, van den Kieboom R, Raymond Y, et al. Aand B-type lamins are differentially expressed in normal human tissues. Histochem Cell Biol. 1997;107(6):505-17.

29 Lindenboim L, Zohar H, Worman HJ, Stein $\mathrm{R}$. The nuclear envelope: target and mediator of the apoptotic process. Cell Death Discov. 2020;6:29.

30 Bell ES, Lammerding J. Causes and consequences of nuclear envelope alterations in tumour progression. Eur J Cell Biol. 2016; 95(11):449-64.

31 Shah P, Wolf K, Lammerding J. Bursting the bubble: nuclear envelope rupture as a path to genomic instability? Trends Cell Biol. 2017; 27(8):546-55.

32 Baylin SB, Jones PA. Epigenetic determinants of cancer. Cold Spring Harb Perspect Biol. 2016;8(9):a019505.

33 Weinberg RA. The biology and genetics of cells and organisms. The biology of cancer. 2nd ed. New York, NY: Garland Science; 2014. p. 1-29.

34 Kuhn TM, Capelson M. Nuclear pore proteins in regulation of chromatin state. Cells. 2019;8(11):1414

35 Malhas AN, Vaux DJ. Nuclear envelope invaginations and cancer. Adv Exp Med Biol. 2014;773:523-35.

36 Doorbar J, Quint W, Banks L, Bravo IG, Stoler M, Broker TR, et al. The biology and lifecycle of human papillomaviruses. Vaccine. 2012;30(Suppl 5):F55-70.

37 Schiffman M, Doorbar J, Wentzensen N, de Sanjosé S, Fakhry C, Monk BJ, et al. Carcinogenic human papillomavirus infection. Nat Rev Dis Primers. 2016;2:16086.

38 Howley PM. Infectious agents and cancer. In Mendelsohn J, Howley, PM, Israel, MA, Gray, JW, Thompson, $\mathrm{CB}$, editor. The molecular basis of cancer. 4th ed. Philadelphia, PA: Elsevier Saunders; 2015. p. 79-102. 